\title{
Hypoxia increases the tempo of evolution in the peri-necrotic niche in glioblastoma
}

\author{
David Robert Grimes ${ }^{1,2^{*}}$, Robert J. Macauley ${ }^{3}$, Frederick J. Currell ${ }^{1}$, Jacob G. Scott ${ }^{4 \dagger}$, \\ and David Basanta ${ }^{\dagger \dagger}$ \\ ${ }^{1}$ Centre for Advanced Interdisciplinary Radiation Research, Queen's University Belfast, BT7 1NN, UK \\ ${ }^{2}$ Cancer Research UK/MRC Oxford Institute for Radiation Oncology, Gray Laboratory, University of Oxford, Old \\ Road Campus Research Building, Off Roosevelt Drive, Oxford OX3 7DQ, UK \\ ${ }^{3}$ Department of Pathology, H. Lee Moffitt Cancer Center \& Research Institute, Tampa, Florida, USA \\ ${ }^{4}$ Departments of Translational Hematology and Oncology Research and Radiation Oncology, Cleveland Clinic, \\ Cleveland, Ohio, USA \\ ${ }^{5}$ Integrated Mathematical Oncology, H. Lee Moffitt Cancer Center \& Research Institute, Tampa, Florida, USA \\ *d.r.grimes@qub.ac.uk \\ †contributed equally
}

\section{${ }_{14}$ ABSTRACT}

Glioblastoma is a deadly brain tumor characterized in part by the histological finding of pseudopallisading necrosis. The cause of this necrosis can be multifactorial, but the consequences are regions of necrosis and hypoxia interspersed throughout the tumors. This heterogeneity in oxygen availability has significant influence on not only cellular population dynamics but also on treatment response. Further, hypoxia has been strongly correlated with the emergence of metastatic and treatment resistant phenotypes. As well as microenvironmental heterogeneity, glioblastoma is one of a number of cancers which have been shown to be composed of a proliferative cellular hierarchy which includes cells with varying abilities to recapitulate the tumor phenotype - the most extreme of which have been recently labeled cancer stem cells, or tumor initiating cells. The interplay between microenvironmental and tumor heterogeneity can explain glioblastoma's somatic evolution but neither clinical data nor biological models can recapitulate this clinical reality alone. Here we present a computational agent-based model of a tumor growing under the proliferative hierarchy in a heterogeneous domain. Our results show that the tempo of tumor stem cell evolution varies widely within the tumor, and is particularly increased at the peri-anoxic edge. We subsequently challenge this provocative in silico finding through analysis of primary histologic samples taken from patients with glioblastoma stained to elucidate areas of hypoxia and necrosis, and to identify heterogeneity in p53. Our results support the hypothesis that the peri-anoxic ridge increases the stem cell turnover, and that this leads to an increase in the mutational load compared to cells in well oxygenated environments. We develop maps of evolutionary tempo from the histology and find that hypoxia effectively 'warps' evolutionary velocity. Implications of this for both tumor evolution and control in glioblastoma are discussed. We develop maps of evolutionary tempo from the histology and find that hypoxia effectively 'warps' evolutionary velocity. Implications of this for both tumor evolution and control in glioblastoma are discussed.

\section{Introduction}

While genetic aberrations are the engine of cancer's somatic evolution, the tumor micro-environment is the key contributor to the selection process that drives it. The micro-environment of a tumor is made of myriad elements that impact the fitness of the tumor cells, thus selecting for the key cancer phenotypes ${ }^{1-3}$ that characterize tumor progression and determine the patient's prognosis. A key element of this micro-environment is oxygen and, as early as the 1950s, investigations by Gray and colleagues $^{4}$ showed its pivotal role in patient prognosis. Since these initial investigations, ample further evidence has emerged confirming the observation that the oxygenation of a tumor has important implications for patient outcome and treatment response $^{5,6}$. There are two major reasons for this - the first is that poorly oxygenated tumors respond significantly worse to treatment than well-oxygenated tumor regions; and second is that oxygen is a known selection pressure which favors specific aggressive cancer cell phenotypes typified by certain known traits. Most prominent of these traits is the capacity to endure harsh environments, and the ability to migrate beyond the tissue from whence they arose ${ }^{7}$.

Such clones gain the ability to proliferate and survive in hypoxic environments ${ }^{8}$. These factors might suggest that hypoxia can initiate metastasis, though the exact mechanism remains unclear. As metastatic cancer is a leading cause of mortality in the 
western world, it is imperative processes such as these, which may contribute to disease evolution, are properly understood. To date, the interplay of these clones with the micro-environment remains poorly understood.

For these reasons, the oxygen micro-environment is of particular interest, and has been studied extensively in physiology and pathophysiology. In healthy tissue, it is relatively stable and well-supplied. However, tumors tend to have highly heterogeneous microscopic oxygen supply ${ }^{9-12}$, a direct consequence of the chaotic and erratic vasculature encouraged by tumor angiogenesis ${ }^{13-15}$. Improving our understanding of the interplay between the oxygen micro-environment and cancer evolution is of paramount importance to advancing therapy ${ }^{16-19}$, yet it is notoriously difficult to probe this question experimentally.

Mathematical models allow us to explore the consequences of various assumptions, even when empirical observations are difficult to obtain. Where data are available, mathematical models can help inform our understanding of what is observed ${ }^{20,21}$, and further, to understand the spatio-temporal dynamics to which the study of fixed tissue or molecular biology is typically blind. It has become increasingly recognised that a synergistic integration of mathematics and clinical as well as experimental data in oncology yields substantial dividends ${ }^{20}$.

In this work, we integrate clinical observations of spatial and temporal heterogeneity in tumor oxygenation with proliferative cellular heterogeneities assuming a tumor made of cells with different degrees of differentiation. Specifically, we developed an agent-based model, using a hybrid discrete-continuous cellular automata (HCA) approach ${ }^{22}$, of neutral tumor evolution in a proliferative hierarchy. Using this model, we studied the evolutionary dynamics of the cancer stem cells across the heterogeneous tumor and find altered evolutionary tempo correlated with areas of extreme hypoxia. Given the strong observations of these intratumoral and microenvironmental heterogeneities in glioblastoma, we test our model derived hypotheses against histology from patients with this disease.

\section{Materials and Methods}

\section{Model outline}

To explore stem cell dynamics, we used an agent-based HCA model built upon the framework we developed previously ${ }^{23}$ with modification. The general schematic for the this is shown in Figure 1. Under this model, stem cells can symmetrically divide (with probability $\alpha$ ), or asymmetrically into a stem daughter and a daughter transient amplifying cell (TACs) with probability $1-\alpha$, provided there is free space for the cells to occupy. While stem cells are effectively immortal unless killed by anoxia, TACs divide to other TACs only, and these cells can only undergo $\beta$ divisions before under-going apoptosis. TAC cell daughters inherit the divisional age of their parent TAC. This framework as described assumes the cancer stem cell hypothesis, essentially the presumption that carcinogenic cells with properties analogous to normal stem cells exist, and can either produce other stem cells or differentiate to other cell types. Typically these CSCs are considered immortal, and the only cells able to give rise to new tumors. The generality and exact properties of cancer stem cells is heavily debated, but has been clearly demonstrated in some cancers such as acute myeloid leukemia (AML) ${ }^{24}$ as well many solid tumor types, including colon ${ }^{25}$, brain $^{26,27}$, breast $^{28}$ and melanoma ${ }^{29}$. In practice, these cells have been hard to find in other tumor types, perhaps due to their relatively low abundance - for AML, cancer stem cell frequency is about 1 in 10,000. An alternative explanation is that any cancer cell can give rise to another cancer cell. Mathematical modelling suggests that the assumptions made will have serious implications for tumor growth ${ }^{30}$, and it is worthwhile to consider both options. To implement the assumption that all cells would proliferate, the simulation was also run with $\alpha=1$ so no TAC cells would emerge. To factor in the influence of the oxygen micro-environment, these simulations were run with heterogeneous oxygen maps, with the addition of conditions for hypoxia mediated death. For cells below a critical oxygen threshold $p_{C}$, cells have a probability $\lambda$ of death per time-step.

The probability of reproduction for a cell, $P_{R}$, is related to available oxygen. As literature suggests, cells are still capable of mitosis down to very low levels of oxygen $\left(\leq 0.5 \mathrm{mmHg}^{31,32}\right.$. The most simple model to capture this reality is a function analogous to a Heaviside step function, where $P_{R}$ stepped to zero below the threshold value. A alternative and perhaps more biologically realistic option was to introduce a function where $P_{R}$ falls with oxygen pressure - a suitable rapidly decaying function which yields $P_{R}=0.5$ at oxygen threshold is given by

$$
P_{R}=1-\exp (-2 \log (2) p)=1-e^{-k p}
$$

where $p$ is oxygen partial pressure and $k=1.368 \mathrm{mmHg}^{-1}$, with the constant chosen such that $P_{R}(0.5)=0.5$. Both possibilities were simulated in this work. Realistic heterogeneous oxygen maps were taken from literature ${ }^{13,15}$ as illustrated in Figure 2 and discussed in the next section. The CA model was run on these oxygen maps, which were presumed to be constant, and we followed the evolution of cancer cells in the micro-environment, recording not only cell position but the divisional age of cells (i.e. the number of total divisions in their life history). This divisional age is taken as a proxy for mutational risk, as cells which undergo more divisions have increased chance of producing an offspring with a clinically relevant mutation, perhaps conferring increased therapeutic resistance or metastatic potential. Each grid position was assumed to be the width of one-cell, and for simplicity no cellular compression was assumed. Parameter values are shown in Table 1. 


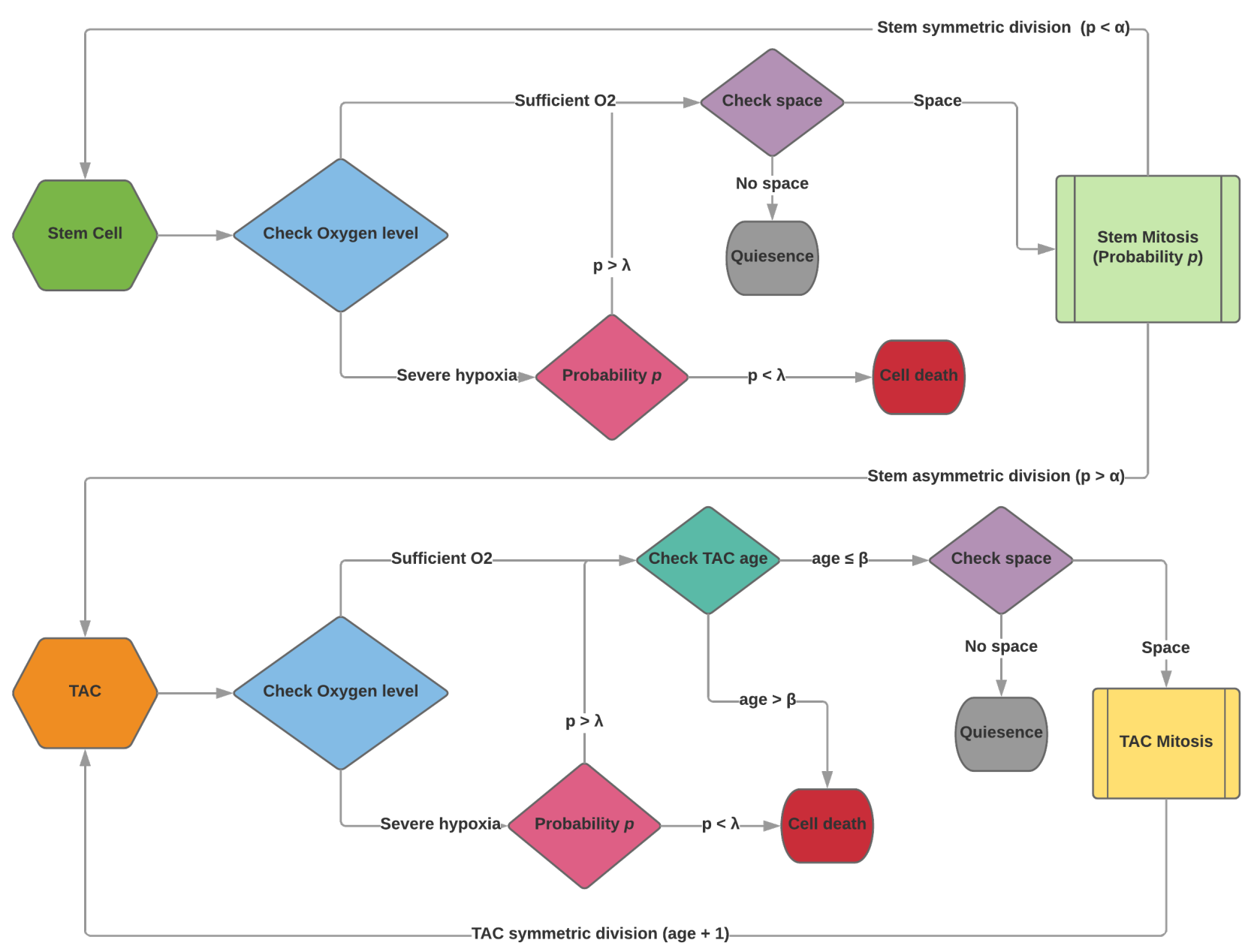

Figure 1. Schematic representation of cellular automata model

\section{Model implementation and Oxygen maps}

The chief goal of this paper is to investigate the role of oxygen in driving somatic evolution in glioblastoma. Accordingly, for simulation, appropriate oxygen maps are required. In tumors, blood vessels provide oxygen to the respiring mass. It has previously been shown that oxygen diffusion distance $\left(r_{n}\right)$ from a vessel or source is limited by the oxygen consumption rate $a$ in both spherical ${ }^{34,35}$ and cylindrical geometries ${ }^{36,37}$. If the underlying vessel geometry is known, it is possible to use previously established methods ${ }^{15}$ to generate estimated oxygen maps in 3D. Due to the complexities of establishing vessel maps by confocal microscopy or others, the sections for which we have complete vessel maps tend to be relatively small (typically $<1 \mathrm{~mm}^{2}$ ). To simulate the heterogeneous nature of tissue oxygen for this work, previously measured and simulated oxygen micro-environments were scaled up to provide different oxygen grids. As the model is employed in two dimensions in this work, 2D slices derived from 3D oxygen maps were used in simulation. The simplest of these corresponded to the oxygen field surrounding a single long vessel, over an area of $1 \times 1 \mathrm{~mm}^{2}$. The second map was chosen to depict an extremely hypoxic micro-environment, derived from a 15 vessel network by ${ }^{13}$, with an area of $5 \times 5 \mathrm{~mm}^{2}$. Finally, a cross-section of a previously described 357 vessel MC-38 mouse tumor ${ }^{15}$ was scaled up and interpolated to correspond to a highly heterogeneous large region of $10 \times 10 \mathrm{~mm}^{2}$. These are depicted in Figure 2. It is important to note that these oxygen maps were assumed to be constant in time, whereas oxygenation is dynamic in real tumors ${ }^{5}$. The separation of time scales between oxygen diffusion and cell division allow making the assumption that the oxygen tension is at a quasi-steady state reasonable ${ }^{37}$. Our model in place, we next sought to quantify whether stem cell division was more prevalent under hypoxic conditions, and test the hypothesis that areas of hypoxia influenced the rate of evolution by changing the rate of stem cell mutation over time. or similar? To investigate this, a single stem cell was seeded in the most oxygen rich environment of each oxygen map at time 0 , and then evolved according to the rules outlined previously. For the single vessel oxygen map, the simulation was run for 2,000 time 
Table 1. Parameter values used in simulation

\begin{tabular}{lcr}
\hline Parameter & Value & Source \\
\hline Critical oxygen threshold $p_{C}$ & $0.5 \mathrm{mmHg}$ & 31 \\
Symmetric stem division probability $\alpha$ & 0.25 & Model parameter \\
TAC division limit $\beta$ & 6 & 33 \\
Hypoxia death probability $\lambda$ & 0.50 & Model parameter \\
Cell diameter & $12.5 \mu \mathrm{m}$ & 32,34 \\
\hline
\end{tabular}

steps to allow cells to grow along the available area. For the 15 vessel oxygen map, simulations were run for 5,000 time steps and finally for the 357 vessel section the simulation was run for 20,000 time steps due to its much greater size. After the simulations were complete, stem cells were stratified by lifetime, relative to the TAC lifetime $\beta$. These were sorted into either high divisions (Number of divisions $>\beta$ ) or low divisions (Number of divisions $\leq \beta$ ). The oxygen levels underlying these stratifications were then compared to determine whether oxygen status significantly skewed distribution.

\section{Experimental data and image analysis}

Human glioblastoma sections were obtained from patient biopsy samples.

For each tumor, three adjacent sections were prepared as follows: 1) hematoxylin and eosin; 2) immunohistochemistry (IHC) for the proliferation marker Ki-67; and 3) IHC for p53 protein, over expression of which is commonly used as a surrogate for TP53 gene mutation. The latter is commonly used as a proxy for mutation, strongly correlated with gene dysregulation in a number of cancers ${ }^{38-42}$. It is important to note that IHC staining alone may not detect all known alterations in the p53 gene in human malignancies ${ }^{43}$, but should suffice to indicate increased mutations where staining is positive. This study was approved by the Moffitt Cancer Center IRB. Microscopy was performed at high resolution using the Digital Pathology Leica Biosystems Aperio system. Images were taken at 20X magnification, yielding digital images of the sections with 1 pixel corresponding to $0.504 \mu \mathrm{m}$. Regions of necrosis were identified by histological examination on the H\&E slide, and marked by a specialized neuro-pathologist (RM) using the Aperio Imagescope software. These annotations could then be extracted as XML files with the coordinates of necrotic boundaries.

As scanned slides were not always properly aligned, a co-registration algorithm for the images was performed. Once images were co-registered, cells both positive and negative for Ki-67 were identified automatically on the Ki-67 slide, as well as P53 positive cells. Finally, the distance from the coordinates of each cell center to each point of the identified necrosis is found, and the minimum distance for each cell stored. Examples of the co-registration and cell identification technique are shown in figure 3. While explicit oxygen concentration cannot be determined from this experimental data, necrosis in glioblastoma is strongly associated with hypoxia, and thus distance from the boundary was treated as a proxy for hypoxia. This assumption is justified in more detail in the discussion section. A full description of the image registration algorithm, image analysis protocol and sample code is included in the supplementary materials. 

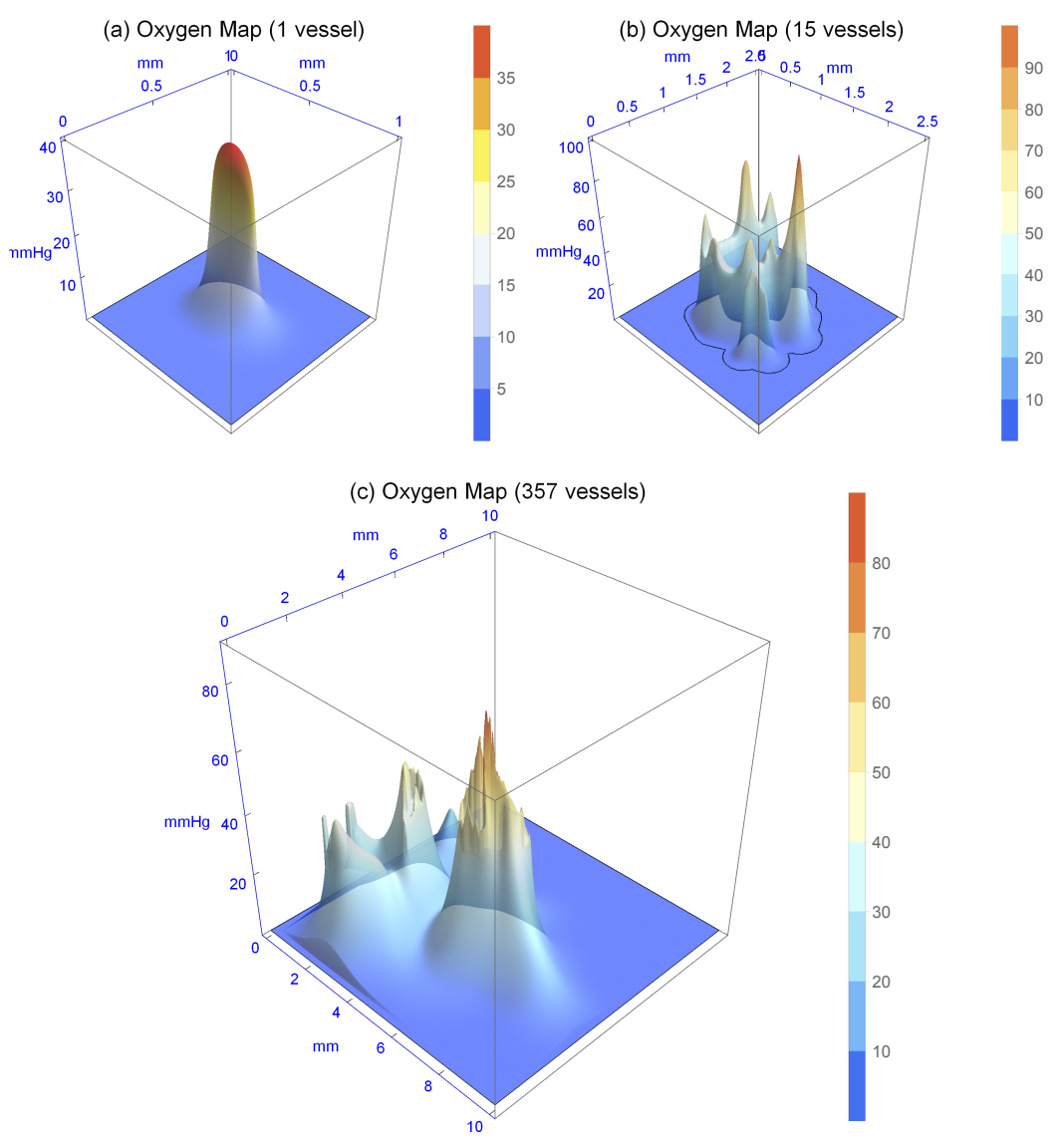

Figure 2. Simulated oxygen maps for this work (a) Oxygen profile from a single vessel (area $1 \mathrm{~mm}^{2}$ ) (b) Oxygen map from a network of 15 vessels (area $25 \mathrm{~mm}^{2}$, adapted from ${ }^{13}$ (c) Oxygen map from a 357 vessel network (area $100 \mathrm{~mm}^{2}$, adapted from ${ }^{15}$.

Results

\section{Simulation results - Divisional rates with oxygen}

The model was run 1,000 times over the oxygen maps outlined and output analysed. Figure 4 depicts the stratification of stem cells in the simulation into two populations (divisions $\leq \beta$ and divisions $>\beta$ ). What is immediately apparent is that high division of stem cells was directly associated with low oxygen conditions. This trend was seen for every map, regardless of the underlying heterogeneity. For all configurations, stem cells on the anoxic border underwent far more divisions than well-oxygenated cells, as illustrated in Figure 5. Qualitative observation of the CA reveal that this increase in divisional age is secondary to cyclic instances of birth and death as cells place daughters into areas of extreme (lethal) hypoxia. So while the daughters are dying, the stem cells continue to divide as they sense free space. With $\alpha=1$ (assuming all cells clonogenic) the same trend was observed, with cells on the anoxic boundary undergoing far more divisions than those in well-oxygenated regions. It should be noted that simulation results were largely insensitive to whether proliferation probability obeyed a simple step-relationship or the decay form shown in equation 1, with negligible difference between both simulation settings.

\section{Analysis of patient data}

From the initial bank of all patient samples, clear borders of necrosis could be ascertained in 23 sections from 9 patients, outlined in Table 2. Sections for analysis ranged from $0.72 \mathrm{~mm}^{2}$ to $108.14 \mathrm{~mm}^{2}$. For each section, image analysis was performed to determine cells that were both positive and negative for Ki-67, and for cells positive for P53 mutations. With these cells and their positions determined, the minimum distance from the cell to the pathologist-specified necrotic boundary was calculated. The probability density for this data is shown in Figure 6, in bins corresponding to the width of two cells $(25 \mu \mathrm{m})$. There was no statistical difference in the distribution of cells both positive and negative for Ki-67 relative to necrosis (two-sample 

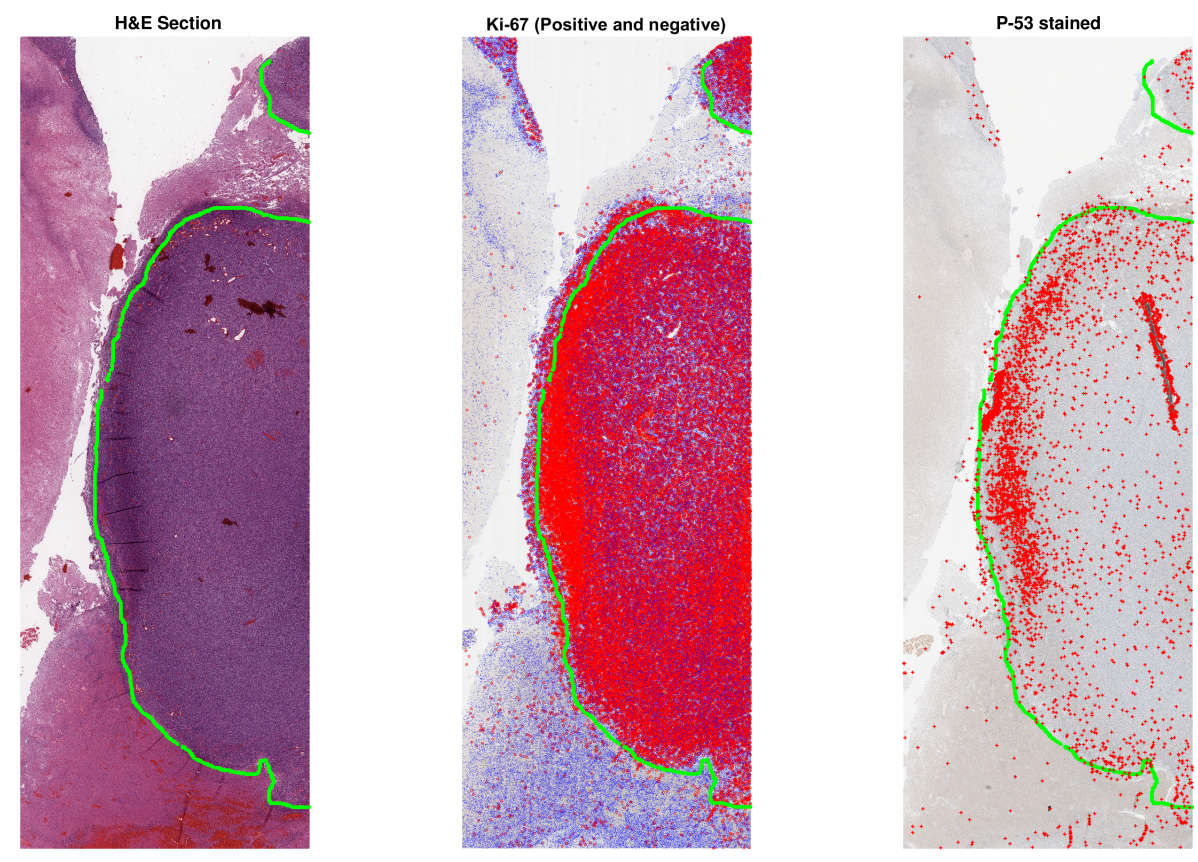

Figure 3. Co-registration and cell detection analysis. A necrotic boundary is marked on the the H\&E slide by the pathologist (marked here by the green line). On the Ki-67 stain, cells which meet the threshold for Ki-67 positive are marked by red dots, and those below threshold by blue dots. Finally, P53 positive cells are marked by red (+) symbols on the final stain. The region shown above encompasses an area of $87.52 \mathrm{~mm}^{2}(15.67 \mathrm{~mm} \times 5.58 \mathrm{~mm}$. )

Kolmogorov-Smirnov test $p=0.5668, \mathrm{KS}$ test statistic 0.0802 ), and accordingly these are grouped together. By contrast, P53 mutation-positive cells are far more likely to be found near regions of necrosis, and have a markedly different distribution than the grouped Ki-67 cells (two-sample Kolmogorov-Smirnov test $p=1.21 \times 10^{-7}$, KS test statistic 0.2941). This suggests an increase in mutational rate associated with hypoxia. This is illustrated clearly in figure 7, which depicts a histological section stained with H\&E. Upon this section, cells straining strongly positive for p53 mutation as detected by the image analysis in the p53 section are superimposed at their corresponding position, marked by blue dots. Regions of clear necrosis as demarcated by the neuropathologist (RM) are outlined in green on this image. From the spatial map of p53 mutant cells, a probability distribution function for these points in space was ascertained by employing a Sheather-Jones data smoothing kernel in Mathematica, which yields the non-parametric probability density function of a random variable ${ }^{44}$. From this, the contour lines of p53 mutation density have been superimposed over the image, with greater line density denoting increased abundance of p53 mutants. For ease of viewing, a red opacity effect has also been superimposed over the image to show the highest density of p53 cells. In this image, the high density of p53 mutant cells tend to lie on or close to the green line of representing the necrotic anoxic boundary, clearly illustrating the increased density of p53 mutants near necrotic boundaries. Under the assumption these necrotic regions are hypoxic, this echoes the phenomena predicted in simulation, yielding a topography of evolutionary velocity. 

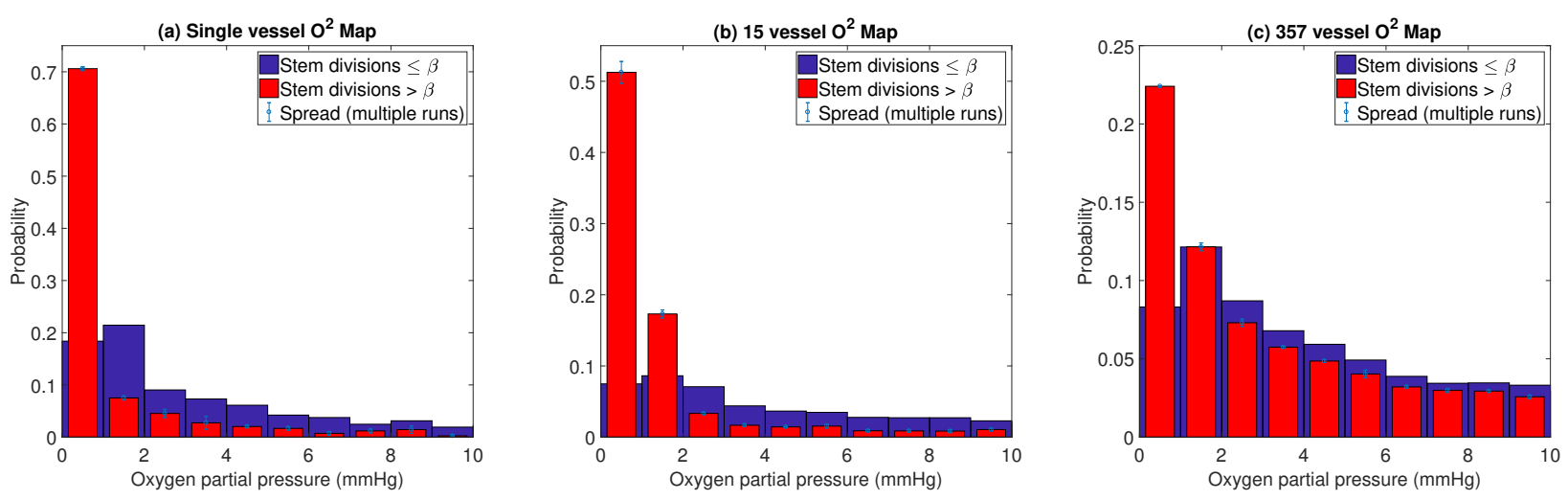

Figure 4. Probability of stem cell division with varying oxygen concentrations. Histograms of stem cell populations in different oxygen environments (only data up to $10 \mathrm{mmHg}$ shown) for (a) single vessel map (b) 15 vessel map and (c) 357 vessel map. Regardless of heterogeneity between maps, the same pattern of higher rates of mitosis in hypoxic conditions was observed.

(a) Stem age

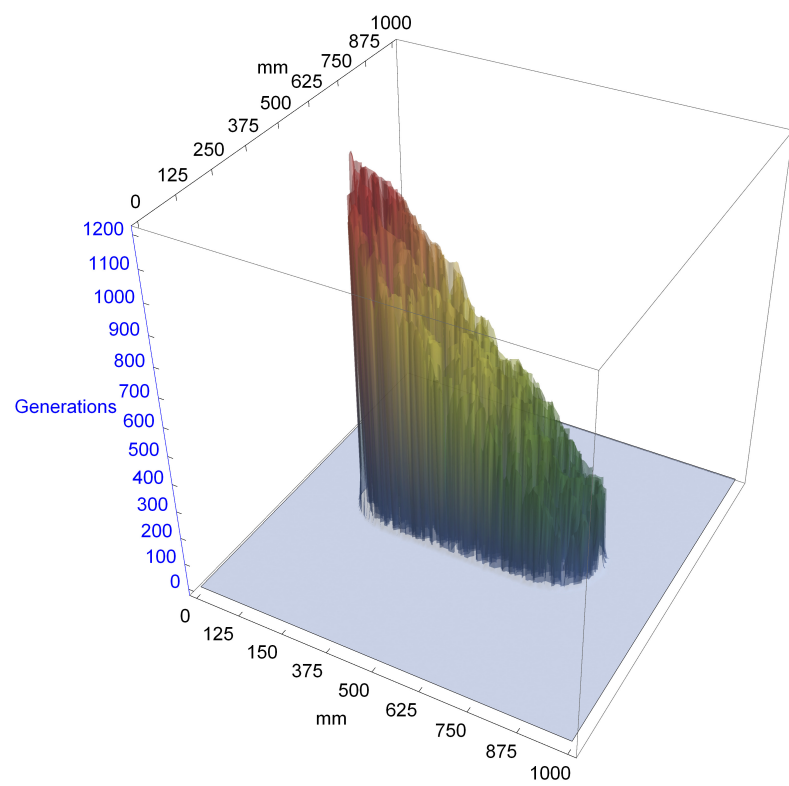

(b) Stem Divisions

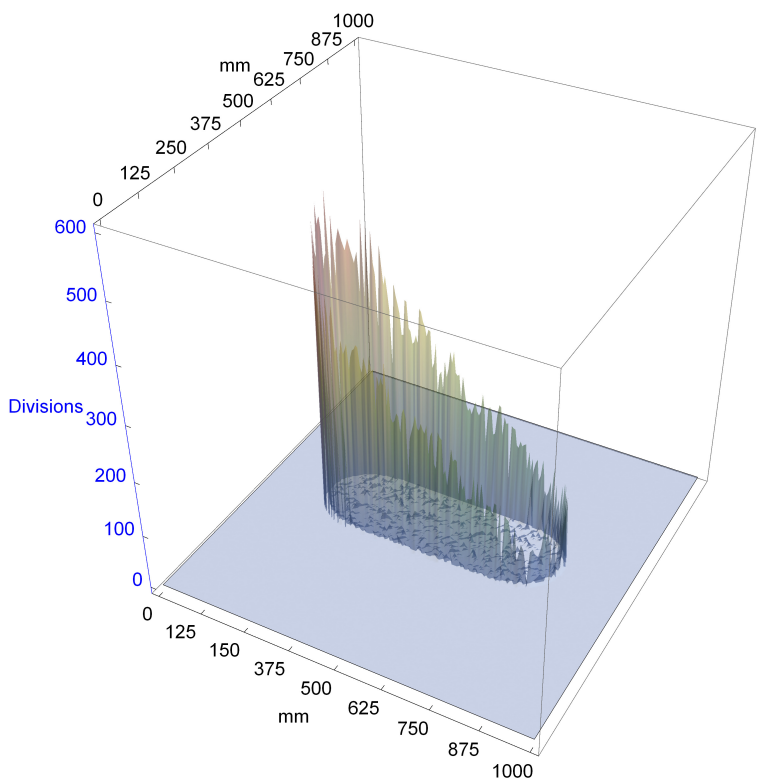

Figure 5. Number of stem cell divisions is correlated chiefly with low oxygen tension rather than stem cell age. (a) Relative ages of stem cells. (b) Number of stem divisions for the same cells. At the anoxic border, mitosis is markedly up-regulated. For this simulation, a vessel-like oxygen map was used with $\beta=4$ and $t=1200$. 
Table 2. Analysis of experimental Glioblastoma sections

\begin{tabular}{lccccr}
\hline Patient sample & Sub-section & Area & Ki-67 + & Ki-67 - & P53 + \\
\hline 1 & i & $108.14 \mathrm{~mm}^{2}$ & 57,498 & 208,789 & 4217 \\
& ii & $87.42 \mathrm{~mm}^{2}$ & 53,435 & 132,068 & 3991 \\
2 & i & $27.21 \mathrm{~mm}^{2}$ & 13,709 & 63,052 & 3072 \\
& ii & $21.10 \mathrm{~mm}^{2}$ & 5814 & 57,689 & 1516 \\
3 & i & $25.82 \mathrm{~mm}^{2}$ & 10,419 & 55,213 & 2722 \\
& ii & $10.08 \mathrm{~mm}^{2}$ & 4794 & 20,108 & 1983 \\
& iii & $18.62 \mathrm{~mm}^{2}$ & 13,580 & 33,186 & 4547 \\
& iv & $15.83 \mathrm{~mm}^{2}$ & 5090 & 53,798 & 275 \\
& v & $4.93 \mathrm{~mm}^{2}$ & 2249 & 14,333 & 120 \\
4 & i & $4.09 \mathrm{~mm}^{2}$ & 2028 & 9569 & 360 \\
5 & i & $2.17 \mathrm{~mm}^{2}$ & 1956 & 4349 & 1676 \\
6 & i & $2.61 \mathrm{~mm}^{2}$ & 1085 & 6125 & 37 \\
& ii & $1.27 \mathrm{~mm}^{2}$ & 500 & 3039 & 33 \\
& iii & $5.18 \mathrm{~mm}^{2}$ & 2736 & 12,905 & 179 \\
& iv & $0.72 \mathrm{~mm}^{2}$ & 385 & 1935 & 7 \\
7 & v & $2.89 \mathrm{~mm}^{2}$ & 943 & 5985 & 252 \\
8 & i & $16.10 \mathrm{~mm}^{2}$ & 14,072 & 39,848 & 4373 \\
& i & $10.88 \mathrm{~mm}^{2}$ & 4275 & 10,845 & 1659 \\
& ii & $26.77 \mathrm{~mm}^{2}$ & 14,406 & 17,757 & 4600 \\
9 & iii & $4.47 \mathrm{~mm}^{2}$ & 2565 & 11,025 & 164 \\
& i & $3.98 \mathrm{~mm}^{2}$ & 1719 & 8090 & 92 \\
& ii & $3.79 \mathrm{~mm}^{2}$ & 1810 & 7930 & 149 \\
& iii & $4.52 \mathrm{~mm}^{2}$ & 1566 & 14,490 & 46 \\
\hline
\end{tabular}



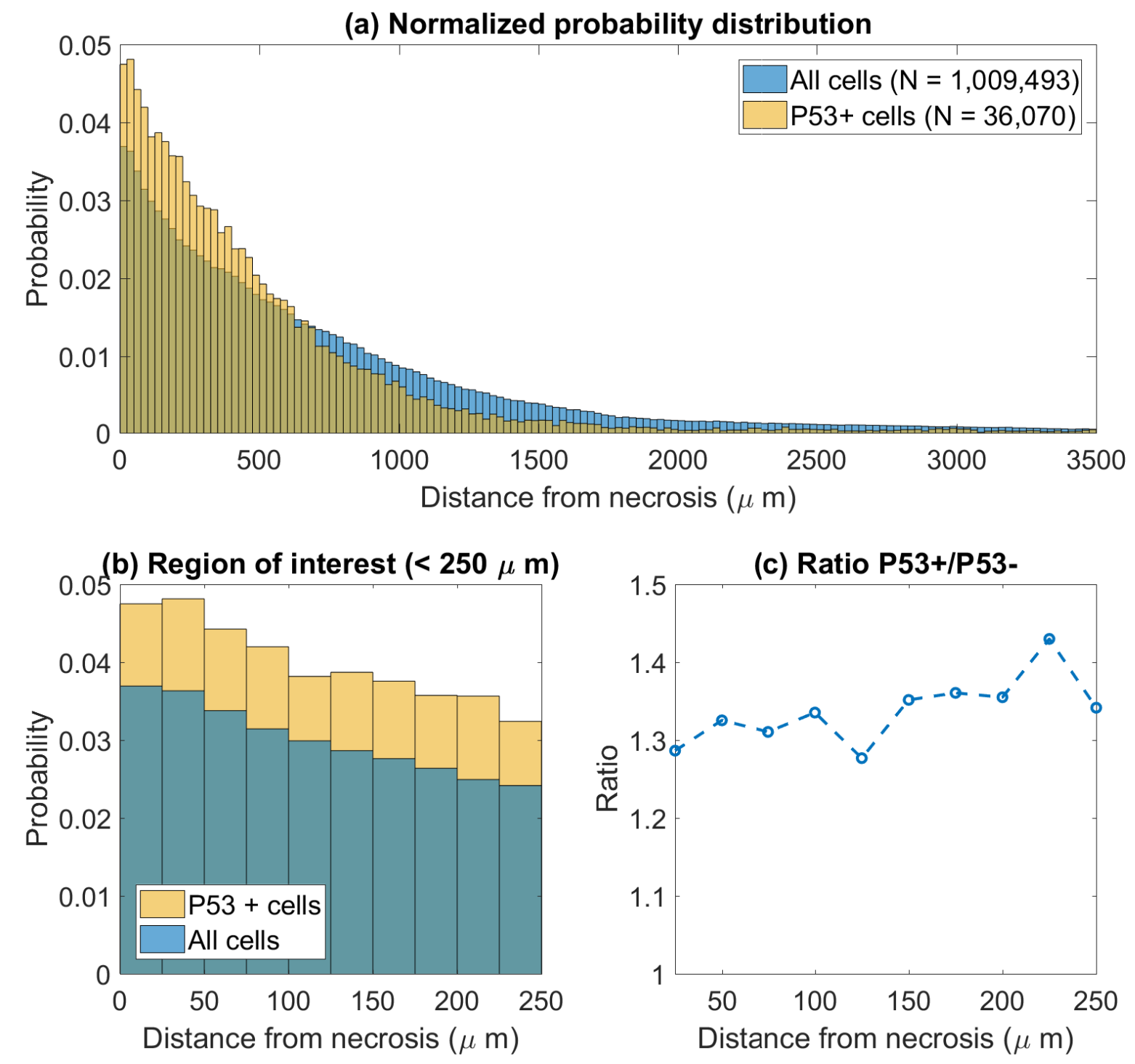

Figure 6. P53 mutations more probable close to known regions of necrosis. Pooled data from 23 regions of 9 patient glioblastoma samples after image analysis. (a) Distribution of P53 stained cells versus Ki-67 stained cells relative to known necrotic borders (b) Probability distributions for stained cells close to necrosis (c) Likelihood Ratio for previous panel. This data suggests that P53-mutations are much more likely to be found close to regions of necrosis and hypoxia, in agreement with model predictions. 
bioRxiv preprint doi: https://doi.org/10.1101/293712; this version posted April 3, 2018. The copyright holder for this preprint (which was not certified by peer review) is the author/funder, who has granted bioRxiv a license to display the preprint in perpetuity. It is made available under aCC-BY-NC-ND 4.0 International license.

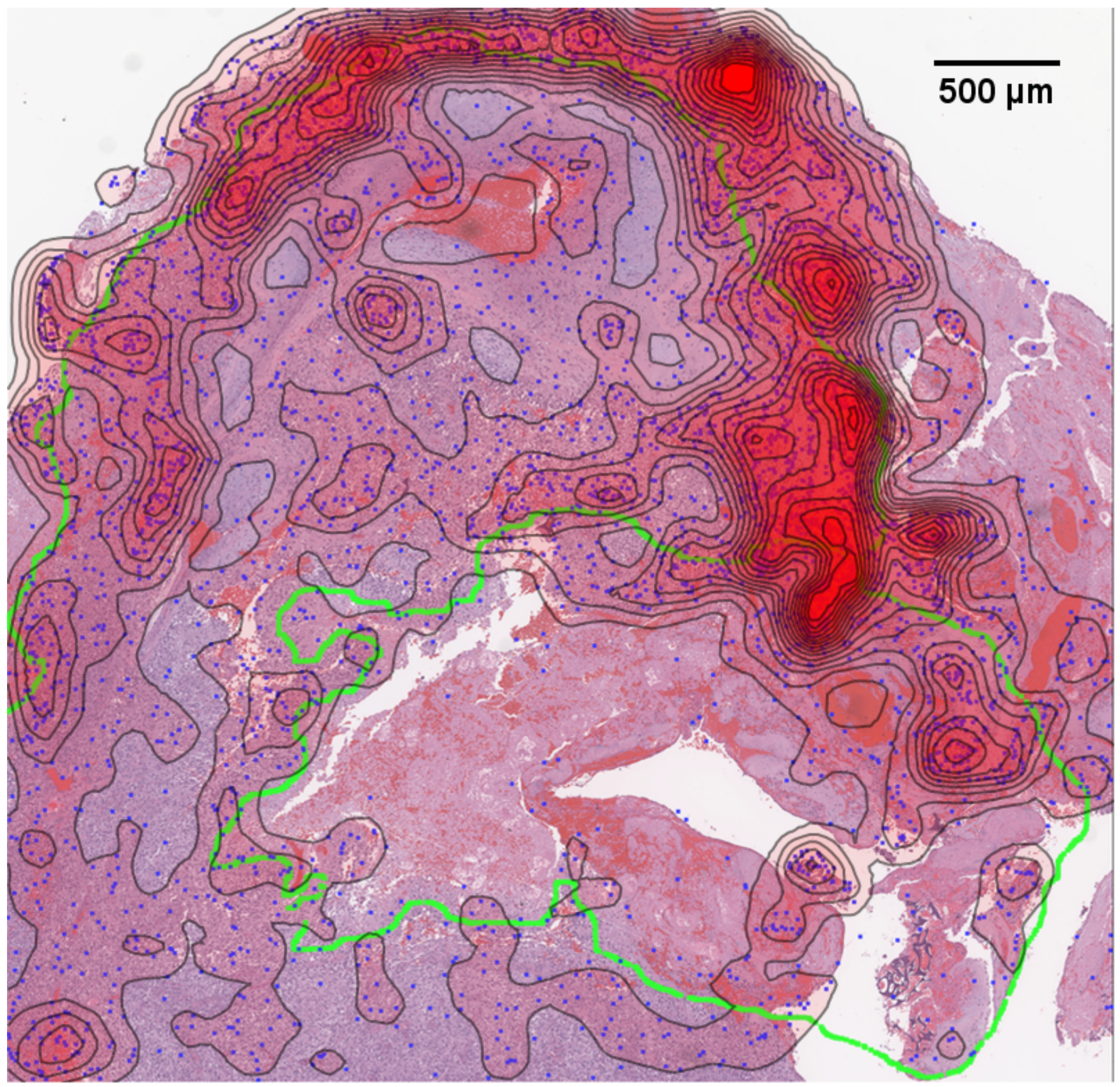

Figure 7. The topography of evolutionary velocity. An example from a patient glioblastoma histologic section. P53 mutations detected by image analysis are illustrated by blue dots overlaid on the histology section. Green lines depict pathologist marked necrosis, and contour lines with red opacity show the probability density of detecting P53 mutations (calculated from a Sheather-Jones smooth kernel distribution function). Near necrotic regions, the probability of finding P53 mutations increases relative to non-necrotic zones. 


\section{Discussion}

Hypoxia and necrosis are known hallmarks of glioblastoma, and literature to date indicates that hypoxia is a selection pressure for aggressive and metastatic phenotypes. Here we have considered the hypothesis that hypoxia can influence the speed and evolutionary potential of glioblastomas, acting as a potential strong selection pressure for subclonal evolution as defined in recent works by fellow researchers ${ }^{45}$. In this work we present evidence that this impact goes beyond selecting for certain phenotypes more adaptable to low oxygen levels, but that hypoxia directly modulates the speed of somatic evolution. Specifically, we show that field representing the speed of somatic evolution is warped near the anoxic edges surrounding areas of necrosis. Mapping the topography of the tumor region reveals marked increase in density of mutant cells in hypoxic domains. To draw an analogy from physics, the presence of hypoxia appears to warp evolutionary velocity in its sphere of influence.

The evidence for this hypothesis was arrived at through two complementary approaches. The first of which was to develop and explore a computational model which focused on how cells in the micro-environment might react to hypoxia. In the computational study we found that stem cell division is substantially more pronounced in regions of hypoxia relative to more well oxygenated regions, as illustrated in figures 4 and 5. It is worth noting too that the findings of this work are in fact largely independent of whether the stem-cell hypothesis is considered or not (data not shown). If we infer that increased stem cell division in the hypoxic niche elevates the probability of a cell acquiring a mutation (and ultimately metastatic potential) then this might in part explain why hypoxia is so strongly correlated with the emergence of metastatic phenotypes and poor prognosis ${ }^{7}$.

The second part of our investigation focused on whether such in silico behaviour might been seen with real tumor sections in situ. To this end, we performed image-analysis on sectioned regions from glioblastoma patients, with co-stained sections. A number of caveats have to be kept in mind when interpreting such data. The most critical of which is that 2D histology is at best an approximation of complex 3D behaviour, and can in some instances be misleading ${ }^{15}$. Another confounding factor was the hardship of defining necrosis robustly - While we had the benefit of a trained neuro-pathologist to demarcate clear regions of necrosis, there were sections which were ambiguous and were left out of the analysis for that reason. This means that the extent of necrosis may in some instances be an underestimate. Even so, a number of suitable sections with clear necrosis were identified in the patient data, with over a million individual cells identified. With such a volume of data, we expect general patterns to become apparent even with the confounding influence of 2D data. P53 mutations were used as a proxy marker for increased mutations, and these were disproportionately seen in regions close to necrosis where oxygen levels are typically extremely low. That mutations were more common in these regions was qualitatively consistent with the hypothesis under investigation, as shown in figure 6 . Figure 7 illustrates this in context, showing the density of p53 mutants is much higher closer to hypoxic zones, depicting the clear 'warping' of evolutionary velocity (defined by number of p53 mutants) in the immediate vicinity of anoxia. It should also be noted that the glioblastoma sections employed in this work had no direct marker of hypoxia. Instead, this work pivots on the implicit assumption that regions of necrosis marked by the clinician were hypoxic. If this assumption is shown to be incorrect, then the experimental correlation between regions of hypoxia and P53 mutation could be called into question. However, even in the absence of a direct metric for hypoxia in these sections there are a number of reasons to suspect necrosis and hypoxia are correlated in glioblastoma. Oxygen-diffusion limited hypoxia giving rise to hypoxia has long been observed in human tissue ${ }^{46}$ and experimental models ${ }^{47}$. There is also a known reciprocal relationship between p53 and hypoxic path ${ }^{48}$. In glioblastoma research specifically there is ample evidence that regions of necrosis are hypoxic ${ }^{49-54}$. Pseudopalisading necrotic cells in particular are known to be hypoxic, displaying dramatic up-regulation of hypoxia inducible factor- $1^{54}$. CA-IX immunostaining was also performed on some of the cases in this work, which confirmed the perinectroic regions were indeed hypoxic. While the necrotic borders in this work are almost certainly hypoxic, direct measurement of the oxygen gradient in the non-anoxic regions was beyond the scope of this work. For future investigations, the ability to quantify the oxygen gradient may yield further insight into the implications for evolution.

In the model presented, cells can either be killed off in the hypoxic zones or in the case of TACS, apoptose after $\beta$ divisions. This prompts the question of whether small amounts of random death might chance the trends observed. To test this, the simulations were also run with random death. For biologically reasonable estimates of this, results were similar with that presented here, as illustrated in supplementary material S1. It's important to note that feature detection in image analysis isn't a simple binary process. Cells over-expressing p53 may do so to different degrees. While strongly positive p53 cells implied mutation, there is a potential that cells staining weakly positive may be due to physiological up-regulation. In this case, for results to be robust it is important to check the same trend is observed when the threshold is modified. As depicted in supplementary $\mathbf{S 1}$, even with modified thresholds p53 straining was more common in perinectrotic regions, increasing confidence that observed effect in this work was not a mere artifact. Image analysis code is included in supplementary $\mathbf{S 2}$.

This work presents combined modelling and experimental evidence that the oxygen micro-environment plays a fundamental role in 'warping' the evolutionary velocity of cells under its influence. In a recent work, Sottoriva and co-author ${ }^{55}$ borrowed the physics analogy of the big bang to illustrate cancer growth as resulting from a single expansion of intermixed clones. The implication of this is that the early origins of the malignancy can be inferred from the current tumor make-up, analogous to determining the origins of the universe from cosmic background radiation. To borrow another rough analogy from cosmology, 
this work presents initial evidence that tumor hypoxia functions like a black hole, bending evolutionary time around the event horizon of an anoxic edge. While this hypothesis requires dedicated experimental investigation, the work outlined here strongly suggests that hypoxia moderates the tempo of evolution in a spatially heterogeneous manner, and is a of critical importance in understanding tumor evolution.

\section{Acknowledgments}

DRG and FJC would like to thank Queen's University Belfast for funding the work of CAIRR. The authors would also like to thank the Integrated Mathematical Oncology department at the H. Lee Moffitt Cancer Center and Research Institute. DRG acknowledges the contributions of NVIDIA research for their generous hardware donations whilst DB acknowledges the National Institute of Cancer (NCI) for grant U01CA202958-01. JGS is grateful to the NIH Loan Repayment program.

\section{Author contributions statement}

DRG, JGS and DB conceived the hypothesis and performed initial tests. DRG and JGS coded the models. RJM performed the histological analysis, and informed the biological discussions. FJC provided institutional support and advice. DRG undertook image analysis. DRG, JGS and DB wrote the manuscript. All authors reviewed the manuscript.

\section{Additional information}

The authors declare no competing interests or conflicts of interest. Code for this manuscript is available in supplementary material.

\section{References}

1. Basanta, D. \& Anderson, A. R. Exploiting ecological principles to better understand cancer progression and treatment. Interface focus 3, 20130020 (2013).

2. Scott, J. \& Marusyk, A. Somatic clonal evolution: A selection-centric perspective. Biochimica et Biophys. Acta (BBA)Reviews on Cancer 1867, 139-150 (2017).

3. Prabhu, A., Kesarwani, P., Kant, S., Graham, S. F. \& Chinnaiyan, P. Histologically defined intratumoral sequencing uncovers evolutionary cues into conserved molecular events driving gliomagenesis and intratumoral heterogeneity in gbm. Neuro-Oncology (2017).

4. Gray, L. H., Conger, A., Ebert, M., Hornsey, S. \& Scott, O. The concentration of oxygen dissolved in tissues at the time of irradiation as a factor in radiotherapy. The Br. journal radiology 26, 638-648 (1953).

5. Grimes, D. R., Warren, D. \& Warren, S. Hypoxia imaging and radiotherapy: bridging the resolution gap. The Br. J. Radiogr. 90, 20160939 (2017).

6. Barker, H. E., Paget, J. T., Khan, A. A. \& Harrington, K. J. The tumour microenvironment after radiotherapy: mechanisms of resistance and recurrence. Nat. Rev. Cancer 15, 409 (2015).

7. Sullivan, R. \& Graham, C. H. Hypoxia-driven selection of the metastatic phenotype. Cancer Metastasis Rev. 26, 319-331 (2007). DOI 10.1007/s10555-007-9062-2.

8. Rankin, E. B., Nam, J.-M. \& Giaccia, A. J. Hypoxia: Signaling the metastatic cascade. Trends Cancer (2016).

9. Shibuya, K. et al. High magnification bronchovideoscopy combined with narrow band imaging could detect capillary loops of angiogenic squamous dysplasia in heavy smokers at high risk for lung cancer. Thorax 58, 989-995 (2003).

10. Carmeliet, P. \& Jain, R. K. Angiogenesis in cancer and other diseases. nature 407, 249-257 (2000).

11. Alarcón, T., Byrne, H. \& Maini, P. A cellular automaton model for tumour growth in inhomogeneous environment. $J$ Theor Biol 225, 257-274 (2003).

12. Baluk, P., Hashizume, H. \& McDonald, D. M. Cellular abnormalities of blood vessels as targets in cancer. Curr. opinion genetics \& development 15, 102-111 (2005).

13. Secomb, T. W., Hsu, R., Dewhirst, M., Klitzman, B. \& Gross, J. Analysis of oxygen transport to tumor tissue by microvascular networks. Int. J. Radiat. Oncol. Biol. Phys. 25, 481-489 (1993).

14. Alarcón, T., Byrne, H. \& Maini, P. A mathematical model of the effects of hypoxia on the cell-cycle of normal and cancer cells. J Theor Biol 229, 395-411 (2004). 
15. Grimes, D. R. et al. Estimating oxygen distribution from vasculature in three-dimensional tumour tissue. J. The Royal Soc. Interface 13, 20160070 (2016). DOI 10.1098/rsif.2016.0070.

16. Scott, J., Fletcher, A., Anderson, A. \& Maini, P. Spatial metrics of tumour vascular organisation predict radiation efficacy in a computational model. PLoS Comput. Biol 12, e1004712 (2016).

17. Grogan, J. et al. Predicting the influence of microvascular structure on tumour response to radiotherapy. IEEE Trans Biomed Eng 9 (2016).

18. Gomes, A. et al. Oxygen partial pressure is a rate-limiting parameter for cell proliferation in $3 \mathrm{~d}$ spheroids grown in physioxic culture condition. PloS one 11, e0161239 (2016).

19. Leek, R., Grimes, D. R., Harris, A. L. \& McIntyre, A. Methods: using three-dimensional culture (spheroids) as an in vitro model of tumour hypoxia. In Tumor Microenvironment, 167-196 (Springer, 2016).

20. Anderson, A. R. \& Quaranta, V. Integrative mathematical oncology. Nat. reviews. Cancer 8, 227 (2008).

21. Altrock, P. M., Liu, L. L. \& Michor, F. The mathematics of cancer: integrating quantitative models. Nat. reviews. Cancer 15, 730 (2015).

22. Anderson, A. R. \& Chaplain, M. Continuous and discrete mathematical models of tumor-induced angiogenesis. Bull. mathematical biology 60, 857-899 (1998).

23. Scott, J. G., Hjelmeland, A. B., Chinnaiyan, P., Anderson, A. R. \& Basanta, D. Microenvironmental variables must influence intrinsic phenotypic parameters of cancer stem cells to affect tumourigenicity. PLoS computational biology 10, e1003433 (2014).

24. Bonnet, D. \& Dick, J. E. Human acute myeloid leukemia is organized as a hierarchy that originates from a primitive hematopoietic cell. Nat. medicine 3, 730-737 (1997).

25. Schepers, A. et al. Lineage tracing reveals Lgr5+ stem cell activity in mouse intestinal adenomas. Sci. 337, 730-5 (2012). DOI 10.1126/science.1224676.

26. Singh, S., Hawkins, C., Clarke, I. \& Squire, J. Identification of human brain tumour initiating cells. Nat. 432, 396-401 (2004).

27. Lan, X. et al. Fate mapping of human glioblastoma reveals an invariant stem cell hierarchy. Nat. (2017).

28. Al-Hajj, M., Wicha, M., Benito-Hernandez, A., Morrison, S. \& Clarke, M. Prospective identification of tumorigenic breast cancer cells. Proc Natl Acad Sci USA 100, 3983-8 (2003). DOI 10.1073/pnas.0530291100.

29. Luo, Y. et al. Aldh1a isozymes are markers of human melanoma stem cells and potential therapeutic targets. Stem Cells 30, 2100-13 (2012). DOI 10.1002/stem.1193.

30. Werner, B. et al. The cancer stem cell fraction in hierarchically organized tumors can be estimated using mathematical modeling and patient-specific treatment trajectories. Cancer research 76, 1705-1713 (2016).

31. Hockel, M. \& Vaupel, P. Tumor hypoxia: definitions and current clinical, biologic, and molecular aspects. J. Natl. Cancer Inst. 93, 266-276 (2001).

32. Grimes, D. R. et al. The role of oxygen in avascular tumor growth. PLoS ONE 11, 1-19 (2016). DOI 10.1371/journal.pone.0153692.

33. Enderling, H. et al. Paradoxical dependencies of tumor dormancy and progression on basic cell kinetics. Cancer research 69, 8814-8821 (2009).

34. Grimes, D. R., Kelly, C., Bloch, K. \& Partridge, M. A method for estimating the oxygen consumption rate in multicellular tumour spheroids. J. Royal Soc. Interface / Royal Soc. 11, 20131124 (2014). DOI 10.1098/rsif.2013.1124.

35. Mueller-Klieser, W. Method for the determination of oxygen consumption rates and diffusion coefficients in multicellular spheroids. Biophys. journal 46, 343-348 (1984).

36. Tannock, I. F. Oxygen diffusion and the distribution of cellular radiosensitivity in tumours. The Br. journal radiology 45, 515-524 (1972).

37. Grimes, D. R., Fletcher, A. G. \& Partridge, M. Oxygen consumption dynamics in steady-state tumour models. Royal Soc. open science 1, 140080 (2014).

38. Boyle, J. O. et al. The incidence of p53 mutations increases with progression of head and neck cancer. Cancer research $\mathbf{5 3}$, 4477-4480 (1993). 
39. Lehman, T. A. et al. p53 mutations, ras mutations, and p53-heat shock 70 protein complexes in human lung carcinoma cell lines. Cancer research 51, 4090-4096 (1991).

40. Rodrigues, N. R. et al. p53 mutations in colorectal cancer. Proc. Natl. Acad. Sci. 87, 7555-7559 (1990).

41. Thorlacius, S. et al. Tp53 mutations and abnormal p53 protein staining in breast carcinomas related to prognosis. Eur. J. Cancer 31, 1856-1861 (1995).

42. Yemelyanova, A. et al. Immunohistochemical staining patterns of p53 can serve as a surrogate marker for tp53 mutations in ovarian carcinoma: an immunohistochemical and nucleotide sequencing analysis. Mod. pathology 24, 1248 (2011).

43. Casey, G. et al. Dna sequence analysis of exons 2 through 11 and immunohistochemical staining are required to detect all known p53 alterations in human malignancies. Oncogene 13, 1971-1981 (1996).

44. Sheather, S. J. \& Jones, M. C. A reliable data-based bandwidth selection method for kernel density estimation. J. Royal Stat. Soc. Ser. B (Methodological) 683-690 (1991).

45. Sun, R. et al. Between-region genetic divergence reflects the mode and tempo of tumor evolution. Nat. genetics 49, 1015 (2017).

46. Thomlinson, R. \& Gray, L. The histological structure of some human lung cancers and the possible implications for radiotherapy. Br. journal cancer $\mathbf{9}, 539$ (1955).

47. Hirschhaeuser, F. et al. Multicellular tumor spheroids: an underestimated tool is catching up again. J. biotechnology 148, 3-15 (2010).

48. Sermeus, A. \& Michiels, C. Reciprocal influence of the $\mathrm{p} 53$ and the hypoxic pathways. Cell death \& disease 2, e164 (2011).

49. Brat, D. J. et al. Pseudopalisades in glioblastoma are hypoxic, express extracellular matrix proteases, and are formed by an actively migrating cell population. Cancer research 64, 920-927 (2004).

50. Brat, D. J. \& Mapstone, T. B. Malignant glioma physiology: cellular response to hypoxia and its role in tumor progression. Annals internal medicine 138, 659-668 (2003).

51. Semenza, G. L. Hypoxia-inducible factor 1: oxygen homeostasis and disease pathophysiology. Trends molecular medicine 7, 345-350 (2001).

52. Zagzag, D. et al. Expression of hypoxia-inducible factor $1 \alpha$ in brain tumors. Cancer 88, 2606-2618 (2000).

53. Monteiro, A. R., Hill, R., Pilkington, G. J. \& Madureira, P. A. The role of hypoxia in glioblastoma invasion. Cells 6, 45 (2017).

54. Rong, Y., Durden, D. L., Van Meir, E. G. \& Brat, D. J. 'pseudopalisading'necrosis in glioblastoma: a familiar morphologic feature that links vascular pathology, hypoxia, and angiogenesis. J. Neuropathol. \& Exp. Neurol. 65, 529-539 (2006).

55. Sottoriva, A. et al. A big bang model of human colorectal tumor growth. Nat. genetics 47, 209-216 (2015). 